
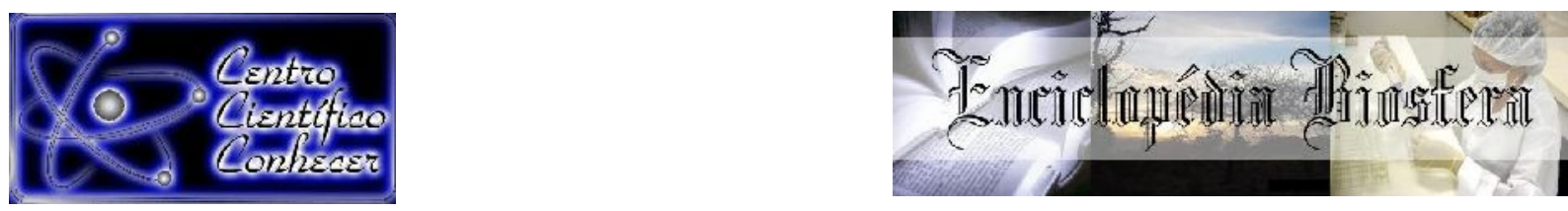

\title{
DETECÇÃO DE RESÍDUOS DE ANTIBIÓTICOS EM PRODUTOS LÁCTEOS
}

\author{
Eloísa dos Santos Siviero ${ }^{1}$ Mariana Luísa Chiezi de Oliveira ${ }^{1}$, Fábio Luiz Bim \\ Cavalieri $^{1,2}$, Isabele Picada Emanuelli ${ }^{1,2}$ \\ ${ }^{1}$ Mestrandas no Programa de Pós-Graduação em Tecnologias Limpas (PPGTL) pela \\ Universidade Cesumar/UNICESUMAR, Maringá-PR - Brasil \\ ${ }^{2}$ Professores Doutores pelo Instituto Cesumar de Ciência, Tecnologia e \\ Inovação/ICETI, Maringá-PR - Brasil \\ E-mail: elosiviero@gmail.com
}

\section{Recebido em: 15/08/2021 - Aprovado em: 15/09/2021 - Publicado em: 30/09/2021 DOI: 10.18677/EnciBio 2021C14}

\begin{abstract}
RESUMO
Este estudo teve por objetivo analisar evidências disponíveis na literatura sobre estudos de triagem de antimicrobianos do tipo antibióticos em leite animal e derivados, visando orientar futuras medidas de saúde pública e melhoria dos processos de produção dos produtos. Trata-se de uma revisão sistemática da literatura realizada nas bases de dados da Plataforma ScienceDirect, PubMed e Scielo. Os critérios de inclusão utilizados foram artigos científicos que identificaram resíduos de antibióticos em leite e produtos lácteos. Foram inclusos estudos publicados na língua portuguesa e inglesa, entre 2005 a 2021. Os critérios de exclusão foram artigos de revisões integrativas, estudos de caso, capítulo de livro e validação metodológica. A busca preliminar identificou 1327 artigos, destes foram selecionados 224 identificados como artigo de pesquisa. Entretanto, a aplicação dos critérios de exclusão e inclusão resultou em apenas 7. Dentre os artigos selecionados a maioria são de Países Europeus, seguido de EUA e China (57,14\%; 28,57\% e 14,29\% respectivamente). Dentre as 2178 amostras de produtos analisadas, $22,75 \%$ testaram positivas para algum dos tipos de antibióticos. Foram identificados 18 tipos de antibióticos nos artigos, sendo as tetraciclinas e $\beta$-lactâmicos os mais presentes nos produtos, configurando respectivamente, $17,24 \%$ e $13,29 \%$. O número de artigos encontrados indicou haver uma escassez de estudos sobre triagem de antimicrobianos em leite animal e derivados. Os resultados evidenciaram que grande parte das amostras de produtos analisadas testaram positivas para algum dos 18 tipos de antibióticos analisados, destacando o risco desses agentes antimicrobianos em alimentos de origem animal para a saúde pública.
\end{abstract}

PALAVRAS-CHAVE: Agentes Antimicrobianos; Contaminantes; Leite Bovino. 


\title{
DETECTION OF ANTIBIOTICS RESIDUES IN DAIRY PRODUCTS: AN INTEGRATIVE REVIEW
}

\begin{abstract}
This study aimed to analyze evidence available in the literature on screening studies of antibiotic-type antimicrobials in animal milk and dairy products, aiming to guide future measures of public health and improvement of the production processes of the products. This is a systematic review of the literature carried out in the databases of the Platform ScienceDirect, PubMed and Scielo. The inclusion criteria used were scientific articles that identified antibiotic residues in milk and dairy products. Studies published in Portuguese and English were included, between 2005 and 2021. Exclusion criteria were integrative review articles, case studies, book chapters and methodological validation. The preliminary search identified 1327 articles, of which 224 were selected as research articles. However, the application of the exclusion and inclusion criteria resulted in only 7. Among the selected articles, most are from European countries, followed by the USA and China $(57.14 \% ; 28.57 \%$ and $14.29 \%$ respectively). Among the 2178 product samples analyzed, $22.75 \%$ tested positive for one of the types of antibiotics. Eighteen types of antibiotics were identified in the articles, with tetracyclines and $\beta$-lactams being the most present in the products, representing respectively $17.24 \%$ and $13.29 \%$. The number of articles found indicated that there is a shortage of studies on antimicrobial screening in animal milk and dairy products. The results showed that a large part of the samples of analyzed products tested positive for one of the 18 types of antibiotics analyzed, highlighting the risk of these antimicrobial agents in foods of animal origin for public health.
\end{abstract}

KEYWORDS: Antimicrobial Agents; Contaminants; bovine milk.

\section{INTRODUÇÃO}

O leite é o principal produto do setor de laticínios e a base para a produção de outros produtos lácteos, destacando-se como uma fonte de proteína animal de alto valor biológico, pois confere fornecimento constante de aminoácidos, auxilia na síntese muscular, beneficia o sistema imunológico e contribui para a saciedade (NOYA et al., 2018; BÄR et al., 2019). Sabendo-se que o leite bovino é um dos ingredientes de origem animal mais consumidos pela população brasileira, torna-se importante o acompanhamento rígido dos padrões de qualidade pela Agência Nacional de Vigilância Sanitária (ANVISA) (MATRASZEK-ZUCHOWSKA et al., 2016 ; WOZNIAK; POSYNIAK, 2016).

Um dos pontos importantes no padrão de qualidade é referente à presença de resíduos farmacológicos no leite. Os animais são medicados com substâncias distintas e cada uma possui sua particularidade, sendo essencial que os médicos veterinários e os funcionários das propriedades sigam às bulas e os períodos de carência determinados (NOVAES et al., 2017). Dentre os medicamentos administrados em vacas leiteiras destacam-se os antibióticos, utilizados nos tratamentos de mastites. Estes medicamentos possuem um amplo espectro de ação para que possam ser eficazes contra os agentes microbianos, entretanto, após sua ação são excretados pelo animal inclusive pela glândula mamária (KAZANCOGLU et al., 2018; OZKAN-OZEN; OZBILTEKIN, 2018). 
O uso indiscriminado e negligente de antimicrobianos em práticas veterinárias, pode favorecer a seleção de genes resistentes na cadeia alimentar humana, causando a resistência bacteriana. Alguns estudos sugerem que a redução do uso de antibióticos na produção animal está associado a uma diminuição de bactérias resistentes a antibióticos em populações humanas (POUPAUD et al., 2021).

A resistência antimicrobiana tornou-se uma ameaça à saúde pública devido à demanda crescente por fontes de alimentos à base de proteína animal, que muitas vezes necessitam utilizar quantidades elevadas de antibióticos durante o processo de criação (SACHI et al., 2019). Para a segurança da saúde pública, os limites máximos de resíduos (LMRs) foram implementados pela Organização para Alimentos e Agricultura. Estes configuram a quantidade máxima de resíduos de antimicrobianos e afins permitida no alimento (FAO, 2018).

Os rebanhos são tratados frequentemente com antibióticos e promotores de crescimento sem acompanhamento veterinário ou especialistas em saúde pública (CHOWDHURY et al., 2012). Assim, a carne de animais de corte, ovo e leite podem conter resíduos de antimicrobianos aumentando a possibilidade de desenvolvimento de bactérias resistentes o que representa um problema de saúde pública (SACHI et al., 2019). O consumo destes produtos alimentícios pelos seres humanos pode acarretar o desenvolvimento de resistência contra antimicrobianos específicos devido à introdução de resíduos antimicrobianos na cadeia alimentar humana (MARSHALL; LEVY, 2011).

É notório que os resíduos de antibióticos estão sendo transferidos para o leite assim como para os produtos lácteos produzidos através dessa matéria prima (FONSECA; SANTOS, 2007). Logo, é essencial por parte dos agricultores seguir os padrões de qualidade determinados pelas agências reguladoras, seguindo às bulas e respeitando os períodos determinados de carência dos antimicrobianos utilizados (NOVAES et al., 2017).

Dentre os setores da pecuária, a cadeia produtiva de leite destaca-se pelos impactos sociais e ambientais, ocasionado pelo manejo sanitário inapropriado das drogas veterinárias do tipo antibiótico que interferem diretamente na qualidade e seguridade dos produtos lácteos (GARCIA et al., 2019; OSBURN; CULLOR, 2019). A interferência na qualidade do produto é ocasionada pela alteração nos processos fermentativos dos produtos como iogurte, queijo e manteiga. Entretanto, os produtores e laticínios parecem estar mais preocupados com os impactos econômicos dos antibióticos nos produtos do que com os problemas ao meio ambiente e à saúde pública como seleção de cepas bacterianas resistentes, no ambiente e no consumidor (QUINTANILLA et al., 2021).

Dentre a repercussão da problemática apresentada, é importante destacar que na análise prévia para investigar a situação de resíduos de antimicrobianos em alimentos lácteos derivados de animais não foi possível evidenciar a real magnitude do problema devido à escassez de estudos de triagem em alimentos. Dessa forma, faz-se necessário uma ampla revisão na literatura científica nacional e internacional para apresentar o cenário atual dos estudos sobre resíduos de antimicrobianos em produtos lácteos.

Diante do exposto, o presente artigo apresenta uma revisão sistemática acerca das evidências disponíveis na literatura sobre estudos de triagem de antimicrobianos 
em leite animal e derivados, visando orientar futuras medidas de saúde pública e melhoria dos processos de produção.

\section{Protocolo de revisão}

\section{MATERIAIS E MÉTODOS}

A revisão seguiu os procedimentos de revisão sistemática padrão, estabelecidos pelos Itens de Relatórios Preferenciais para Revisões Sistemáticas e Meta-Análises (PRISMA) (LIBERATI et al., 2009). A presente revisão sistemática teve o intuito de responder a seguinte questão ainda não descrita na literatura: "Qual o cenário atual dos estudos de resíduos antimicrobiano em produtos lácteos?".

As diretrizes processuais apresentadas na Figura 1 foram seguidas: (a) pesquisa de banco de dados para categorizar artigos potencialmente relevantes, (b) avaliação da relevância dos artigos, (c) avaliação da qualidade e (d) extração de dados. A Figura 1 mostra a estratégia de pesquisa e processo seletivo para artigos de pesquisa publicados entre os anos de 2005 e 2021. Com base nos critérios de pesquisa, foram identificados 1327 artigos publicados que foram analisados e refinados para a seleção dos artigos alvos deste estudo descritos no fluxograma PRISMA.

\section{Estratégia de pesquisa}

Uma abordagem estruturada de busca de literatura foi utilizada para identificar estudos publicados relatando a presença de resíduos de antibióticos em leite de animais e seus derivados. As bases de dados científicas, Scielo (https://www.scielo.org/) Science Direct (https://www.sciencedirect.com/) e PubMed (https://pubmed.ncbi.nlm.nih.gov/) foram pesquisadas por estudos relevantes publicados entre os anos de 2005 e 2021.

Para determinar os termos de busca foi realizada uma pesquisa prévia com alguns termos de busca visando selecionar os termos que mais encontravam artigos científicos nas bases de dados utilizadas. Os termos de busca selecionados e aplicados nas pesquisas desta revisão foram os seguintes: métodos de triagem, antibiótico, leite, resíduos, produtos lácteos (screening methods, milk, antibiotic, Waste, dairy products). $\mathrm{Na}$ base de dados Scielo utilizou-se os termos de busca em português e em inglês, e nas demais bases internacionais apenas em inglês. Não houve duplicata de artigo entre as bases pesquisadas. Foram retirados estudos que não atendiam aos critérios de inclusão predeterminados.

\section{Triagem de dados}

Os textos completos dos artigos publicados recuperados foram selecionados para inclusão. Foram selecionados os estudos para avaliação os artigos que atenderam aos seguintes critérios de inclusão:

- Qualquer artigo de pesquisa publicado entre os anos de 2005 e 2021 que relatava resíduos de antibióticos em leite e produtos lácteos.

- Qualquer artigo de pesquisa que relatasse a prevalência, investigação, incidência, ocorrência, levantamento, caracterização e identificação de resíduos de antibióticos.

- Os dados foram extraídos e registrados para localização do estudo, citação, primeiro autor, tempo de estudo, ano de publicação, tipo de 
amostra, tamanho amostral, número de amostras positivas, presença ou ausência de resíduo antimicrobiano, sensibilidade ou percentual de nível de resíduos de antibióticos e métodos utilizados para detecção.

FIGURA 1. Fluxograma dos Itens de Relatórios Preferenciais para Revisões Sistemáticas e Meta-Análise (PRISMA).
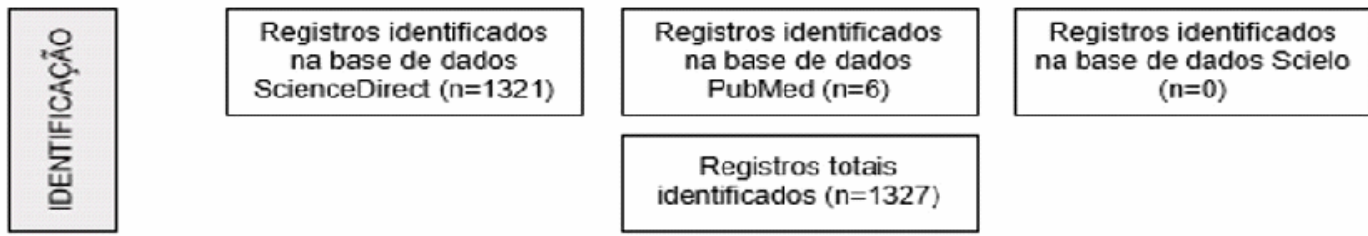

Registros totais identificados $(n=1327)$
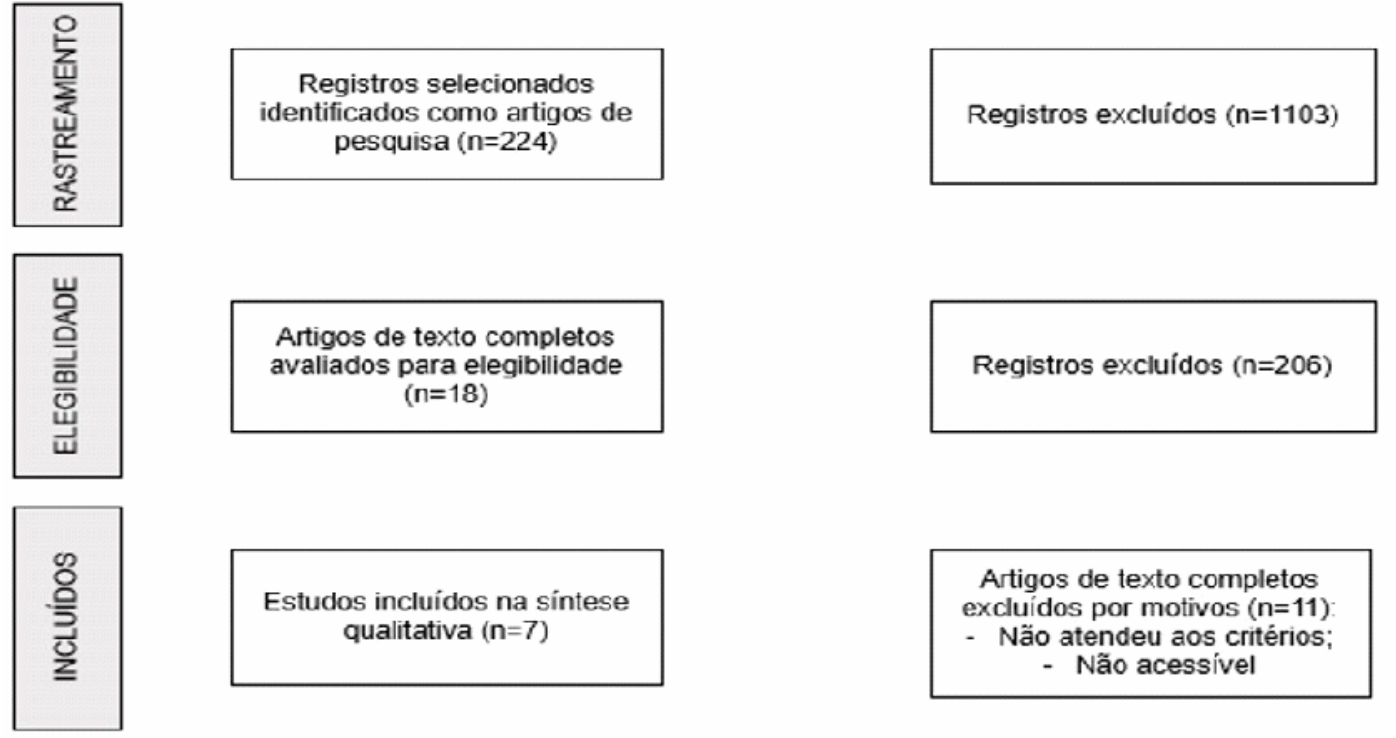

Artigos de texto completos excluídos por motivos $(n=11)$

- Näo atendeu aos critérios: - Não acessivel

Fonte: Autores (2021).

Todos os estudos que se enquadravam nos critérios de exclusão foram descartados da pesquisa. Os critérios de exclusão utilizados foram:

- Qualquer estudo que não seja artigo de pesquisa, como artigo de revisão, estudo de caso, capítulo de livro e validação metodológica.

Os artigos elegíveis foram recuperados em formato de texto completo e avaliados usando as definições de caso especificadas pelos respectivos estudos (Quadro 1).

\section{Análise de dados}

Todos os dados extraídos das diferentes publicações foram classificados no Microsoft Excel® e analisados por estatística descritiva. Os resultados do estudo desses artigos foram classificados de acordo com ano de publicação, região e período dos estudos, origem onde as amostras foram coletadas, as espécies animais utilizadas nos estudos, o tipo de amostra analisada, os métodos analíticos aplicados para quantificação dos resíduos de antimicrobianos, os antibióticos detectados por esses métodos, o total de amostras analisadas e as respectivas amostras positivas. 
QUADRO 1. Detecção, fontes e prevalência de resíduos de antimicrobianos entre 2005 e 2021 nas áreas de estudo.

\begin{tabular}{|c|c|c|c|c|c|c|c|c|c|}
\hline $\begin{array}{l}\text { AUTOR } \\
\text { /ANO }\end{array}$ & $\begin{array}{c}\text { REGIÃO } \\
\text { DO } \\
\text { ESTUDO }\end{array}$ & $\begin{array}{l}\text { PERÍODO } \\
\text { DO } \\
\text { ESTUDO }\end{array}$ & $\begin{array}{l}\text { ORIGEM } \\
\text { DA } \\
\text { COLETA }\end{array}$ & $\begin{array}{l}\text { ESPÉCIE } \\
\text { ANIMAL }\end{array}$ & $\begin{array}{l}\text { TIPO DE } \\
\text { PRODUTO }\end{array}$ & $\begin{array}{l}\text { MÉTODO } \\
\text { ANALÍTICO } \\
\text { APLICADO }\end{array}$ & $\begin{array}{l}\text { ANTIBIÓTICOS } \\
\text { DETECTADOS }\end{array}$ & $\begin{array}{c}\text { AMOSTRAS } \\
\text { POSITIVAS (N) }\end{array}$ & $\begin{array}{c}\text { AMOSTRAS } \\
\text { TOTAIS } \\
\text { ANALISADAS } \\
\text { (N) }\end{array}$ \\
\hline $\begin{array}{l}\text { TASCI } \\
\text { et al., } \\
2021\end{array}$ & $\begin{array}{l}\text { Turquia / } \\
\text { Província } \\
\text { de Burdur }\end{array}$ & $2020-2021$ & $\begin{array}{l}\text { Coletadas } \\
\text { aleatoriam } \\
\text { ente }\end{array}$ & $\begin{array}{l}\text { Vaca e } \\
\text { cabra }\end{array}$ & $\begin{array}{l}\text { colostro, } \\
\text { leite cru e } \\
\text { leite UHT }\end{array}$ & $\begin{array}{l}\text { LC/MS e } \\
\text { ionização por } \\
\text { eletrospray }\end{array}$ & $\begin{array}{c}\text { Tetraciclina, 4- } \\
\text { epitetracycline, } 4- \\
\text { epioxytetracycline, } \\
4- \\
\text { epichlorotetracycli } \\
\text { ne e } \\
\text { ciprofloxacina }\end{array}$ & 72 & 130 \\
\hline $\begin{array}{l}\text { CHIE } \\
\text { SA et } \\
\text { al., } \\
2020\end{array}$ & $\begin{array}{l}\text { Itália / } \\
\text { Região } \\
\text { Norte }\end{array}$ & $2019-2020$ & $\begin{array}{l}\text { Fazendas } \\
\text { leiteiras }\end{array}$ & Vaca & $\begin{array}{l}\text { leite cru e } \\
\text { queijo }\end{array}$ & LC-HRMS & $\begin{array}{c}\text { Lincomicina, } \\
\text { oxitetraciclina, } \\
\text { cefapirina, } \\
\text { espiramicina }\end{array}$ & 35 & 254 \\
\hline $\begin{array}{l}\text { BILAN } \\
\text { DŽIĆ } \\
\text { et al., } \\
2011\end{array}$ & Croácia & $2008-2010$ & $\begin{array}{l}\text { Fazendas, } \\
\text { produtores } \\
\text { e laticínios }\end{array}$ & Vaca & Leite cru & HPLC-DAD & $\begin{array}{l}\text { Clororamfenicol, } \\
\text { penicilinas, } \\
\text { cefalosporinas, } \\
\text { tetraciclinas, } \\
\text { sulfonamidas, } \\
\text { beta-lactams, } \\
\text { quinolones, } \\
\text { aminoglycosides e } \\
\text { macrolides }\end{array}$ & 37 & 1259 \\
\hline $\begin{array}{l}\text { DU et } \\
\text { al., } \\
2019\end{array}$ & China & 2016 & Mercados & Vaca & $\begin{array}{l}\text { Leite UHT e } \\
\text { Leite } \\
\text { pasteurizado }\end{array}$ & $\begin{array}{l}\text { Imunoabsorvente } \\
\text { ligado a enzima } \\
\text { técnica de chip de } \\
\text { microarray }\end{array}$ & $\begin{array}{l}\text { Tetraciclinas, } \\
\text { quinolonas, } \\
\text { lincomicina e } \\
\text { estreptomicina }\end{array}$ & 123 & 198 \\
\hline $\begin{array}{l}\text { TEMP } \\
\text { INI et } \\
\text { al., } \\
2018\end{array}$ & $\begin{array}{c}\text { EUA / } \\
\text { Califórnia }\end{array}$ & 2016-2017 & $\begin{array}{l}\text { Fazendas } \\
\text { leiteiras }\end{array}$ & Vaca & Leite cru & LC/MS & Antibióticos & 15 & 25 \\
\hline $\begin{array}{l}\text { RAND } \\
\text { ALL et } \\
\text { al., } \\
2014\end{array}$ & $\begin{array}{l}\text { Reino } \\
\text { Unido / } \\
\text { Inglaterra } \\
\text { e País de } \\
\text { Gales }\end{array}$ & 2011 & $\begin{array}{l}\text { Fazendas } \\
\text { leiteiras }\end{array}$ & Vaca & Leite cru & LC/MS & $\begin{array}{c}\beta \text {-lactâmicos e } \\
\text { tetraciclinas }\end{array}$ & 66 & 103 \\
\hline $\begin{array}{l}\text { PEREI } \\
\text { RA et } \\
\text { al., } \\
2014\end{array}$ & $\begin{array}{l}\text { EUA / } \\
\text { Centro de } \\
\text { NY }\end{array}$ & 2013 & $\begin{array}{l}\text { Fazendas } \\
\text { leiteiras }\end{array}$ & Vaca & Leite cru & LC/MS & $\begin{array}{c}\beta \text {-lactâmicos, } \\
\text { penicilinas e } \\
\text { cefalosporinas }\end{array}$ & 143 & 189 \\
\hline
\end{tabular}

Cromatografia líquida-espectrometria de massa (LC-MS); Método multiclasse da Espectrometria de Alta Resolução da Cromatografia Líquida tandem Mass (LC-HRMS); Cromatografia líquida com arranjo de diodos (HPLC - DAD).

\section{Aquisição de dados}

\section{RESULTADOS E DISCUSSÕES}

A busca preliminar identificou como registros totais 1327 artigos. A pesquisa manual não identificou artigos adicionais e não houve duplicação de artigos nas bases de dados pesquisadas. Destes artigos foram selecionados 224 registros identificados como artigo de pesquisa. A aplicação dos critérios de exclusão e inclusão resultou em sete artigos que foram identificados como elegíveis para extração de dados e análise qualitativa (Quadro 1). A maioria dos artigos selecionados (217) foi excluída por se tratar de desenvolvimento de métodos analíticos de detecção ou por ser estudos experimentais com grupos tratamentos e não triagem de antibióticos em produtos alimentícios de origem animal. 
Os artigos identificados relatando a presença de resíduos de antibióticos em leite de animais e seus derivados foram pesquisados por estudos relevantes publicados entre os anos de 2005 e 2021. Entre os anos de 2005 a 2010 não houve nenhuma publicação evidenciando resíduos de antibióticos no leite ou seus derivados. Depois de 2011 as publicações anuais mantiveram-se em um artigo ao ano, com exceção do ano de 2014 que, diferente dos demais, obteve dois artigos publicados.

FIGURA 2. Quantidade de artigos publicados por ano com o tema resíduos de antimicrobianos em leite animal e derivados.

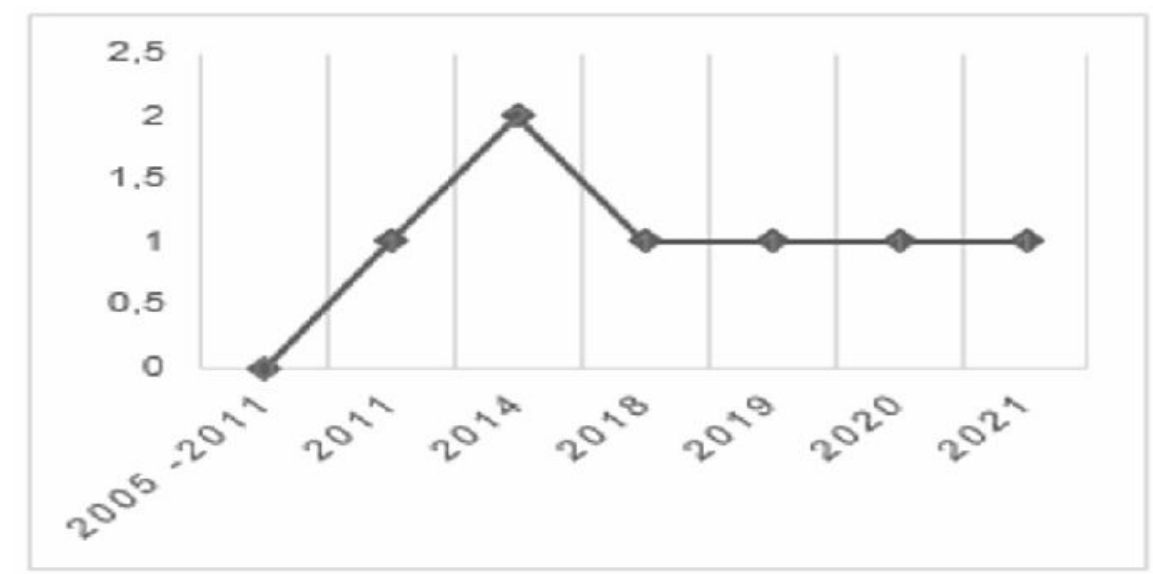

Fonte: Autores (2021).

\section{Região de estudo}

Dentre os artigos selecionados, as localizações geográficas mais comuns e com maior número de publicações foi a Europa com 57,14\% (BILANDŽlĆ et al., 2011; PEREIRA et al., 2014; RANDALL et al., 2014; CHIESA et al., 2020; TASCl et al., 2021; CANBAY; DOGANTURK, 2021) seguido dos EUA com 28,57\% (TEMPINI et al., 2018;) e da China com 14,29\% (DU et al., 2019) (Figura 3A e 3B). Esta análise indicou claramente haver escassez de estudos sobre triagem de antimicrobianos em leite animal e derivados, principalmente na América do Sul. Mesmo sendo utilizadas palavras de busca em português nas pesquisas da base de dados, não foram encontrados artigos sobre o tema em questão na América do Sul, expondo a importância de fomentar estudos no Brasil e na América latina. 
FIGURAS 3A e 3B. Localizações geográficas mais comuns e com maior número de publicações de autores de artigos com o tema resíduos de antimicrobianos em leite animal e derivados.

3A.

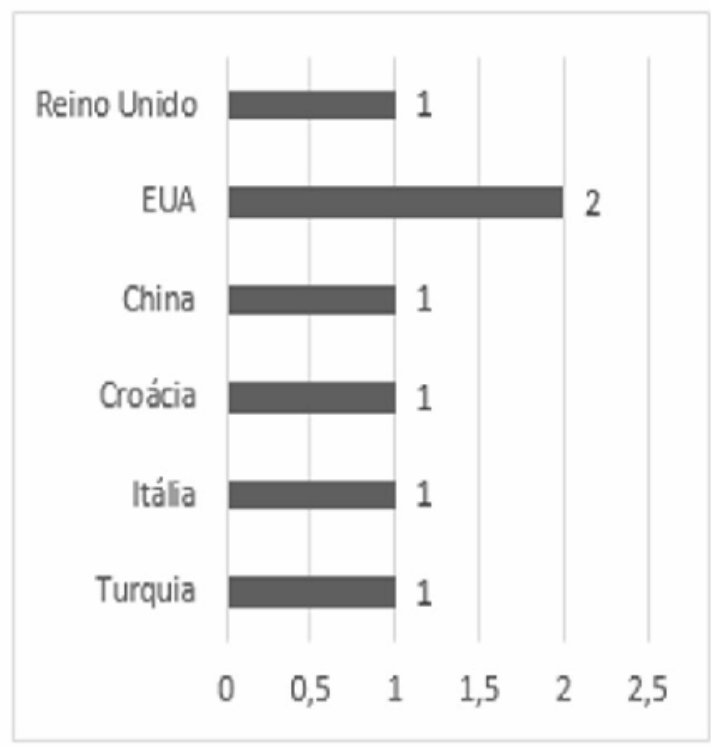

3B.

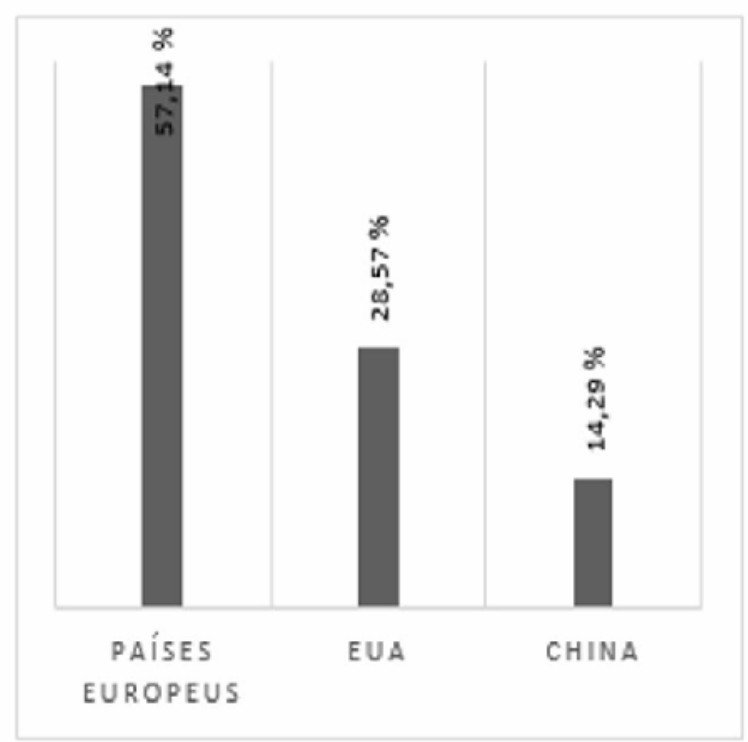

Fonte: Autores (2021).

\section{Espécie animal e tipo de amostras}

As espécies animais que tiveram seus leites e derivados estudados nos artigos foram vaca $(n=7)$ e cabra $(n=1)$. Os tipos de produtos estudados nos artigos de triagem de antimicrobiano foram basicamente leites, colostro e queijos, destes $58,33 \%(n=7)$ foram leite cru, 16,67\% ( $n=2)$ leite UHT (Figura 4). As demais amostras foram leite pasteurizado, colostro e queijo apenas com um estudo cada $(8,33 \% ; n=1)$. Produtos como o leite e seus respectivos derivados são pouco retratados na literatura. Um levantamento bibliográfico realizado em 2015 revelou apenas dois estudos que realizaram triagem de antibióticos em amostras de leite (CHOWDHURY et al., 2015; SACHI et al., 2019). Dentre os estudos realizados para detecção de agentes antimicrobianos em produtos alimentícios, os artigos mais apresentados na literatura são de produtos cárneos (BRISTY et al., 2019; FERDOUS, 2019). 
FIGURA 4. Tipo de produto analisado nos artigos estudados com a temática resíduos de antimicrobianos em leite animal e derivados.

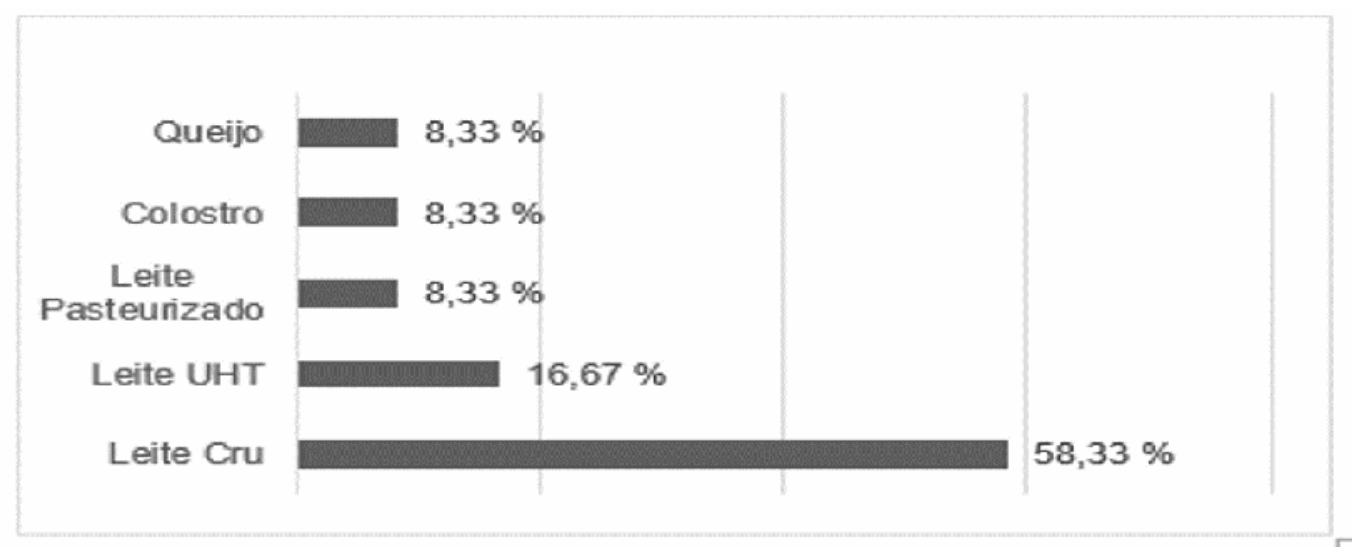

Fonte: Autores (2021).

\section{Métodos analíticos}

Majoritariamente o método analítico mais empregado nas triagens dos estudos para a detecção dos antimicrobianos foi o LC/MS, configurando um total de $57,14 \%$ $(n=4)$ dos artigos estudados (Quadro 2). Outros métodos analíticos de detecção como LC/MS e ionização por eletrospray, LC-HRMS, HPLC-DAD e Imunoabsorvente ligado à enzima técnica de chip de microarray também foram utilizados nas triagens dos estudos. Estes métodos confirmam com o fato apresentado na literatura sobre a utilização da Cromatografia, especialmente o método cromatográfico HPLC, TLC e LC para detecção de antimicrobianos como traciclina, amoxicilina e ciprofloxacina no leite (CHOWDHURY et al., 2015; FERDOUS et al., 2019;).

QUADRO 2. Métodos analíticos empregados nas triagens dos estudos para detecção antimicrobiana dos artigos analisados com o tema resíduos de antibióticos em leite animal e derivados.

\begin{tabular}{|c|c|c|}
\hline Métodos & Número de artigos & Descrição \\
\hline LC/MS & 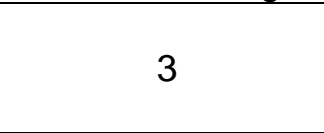 & $\begin{array}{l}\text { Cromatografia líquida } \\
\text { acoplada à espectrometria de } \\
\text { massas }\end{array}$ \\
\hline LC-HRMS & 1 & $\begin{array}{l}\text { Método mutilasses da } \\
\text { Espectrometria de Alta } \\
\text { Resolução da Cromatografia } \\
\text { Líquida tandem Mass }\end{array}$ \\
\hline HPLC - DAD & 1 & $\begin{array}{c}\text { Cromatografia líquida com } \\
\text { arranjo de diodos }\end{array}$ \\
\hline $\begin{array}{l}\text { Imunoabsorvente de } \\
\text { Chip de Microarray }\end{array}$ & 1 & $\begin{array}{l}\text { Imunoabsorvente ligado a } \\
\text { enzima técnica de chip de } \\
\text { microarray }\end{array}$ \\
\hline LC/MS por Eletrospray & 1 & $\begin{array}{l}\text { LC/MS e ionização por } \\
\text { eletrospray }\end{array}$ \\
\hline
\end{tabular}




\section{Agentes antimicrobianos residuais nas amostras}

Os estudos analisados avaliaram um total de 2178 amostras de produtos, sendo que destas $22,75 \%(n=491)$ testaram positivas para algum dos tipos de antibióticos (Quadro 1). Foram identificados nos artigos 18 tipos de antibióticos, sendo as tetraciclinas e os $\beta$-lactâmicos os mais presentes caracterizando $17,24 \%$ e $13,29 \%$, respectivamente (Figura 5). Estes achados corroboram com o fato apresentado na literatura de que dentre os principais antimicrobianos administrados ao rebanho leiteiro, encontram-se os $\beta$-lactâmicos e as tetraciclinas (MEDEIROS et al., 2009; SANI et al., 2010; NIKPOOYAN; MOSHIRI, 2010).

A literatura aponta o grupo dos $\beta$-lactâmicos como o mais difundido entre os antibióticos utilizados no tratamento de infecções em vacas leiteiras, representando em média $38,22 \%$ do total de antibióticos, seguido das tetraciclinas com 15,41\% (SANI et al., 2010; NIKPOOYAN; MOSHIRI, 2010). Essa pesquisa desenvolvida por Sani e colaboradores (2010) é um estudo comparativo entre regiões leiteiras da Espanha, Irã e do Brasil. A triagem realizada na região de Castilla, detectou a presença de $\beta$-lactâmicos em $29,8 \%$ das amostras; no irã índices superiores (40,8\%); já no Brasil, $11,4 \%$ das amostras de leite, provenientes de quatro regiões leiteiras, apresentaram este antibiótico. A literatura também aponta a tetraciclina como um antibiótico de uso frequente em tratamentos de mastites (BRITO et al., 2001; MEDEIROS et al., 2009). A tetraciclina tem ação sobre bactérias Gram-positivas e Gram-negativas, porém deve-se ter cautela em seu uso, pois induz a resistência antimicrobiana plasmidial, podendo carrear resistência para outros antibióticos (MEDEIROS et al., 2009).

FIGURA 5. Agentes antimicrobianos residuais identificados nas amostras analisadas nos artigos com a temática resíduos de antimicrobianos em leite animal e derivados.

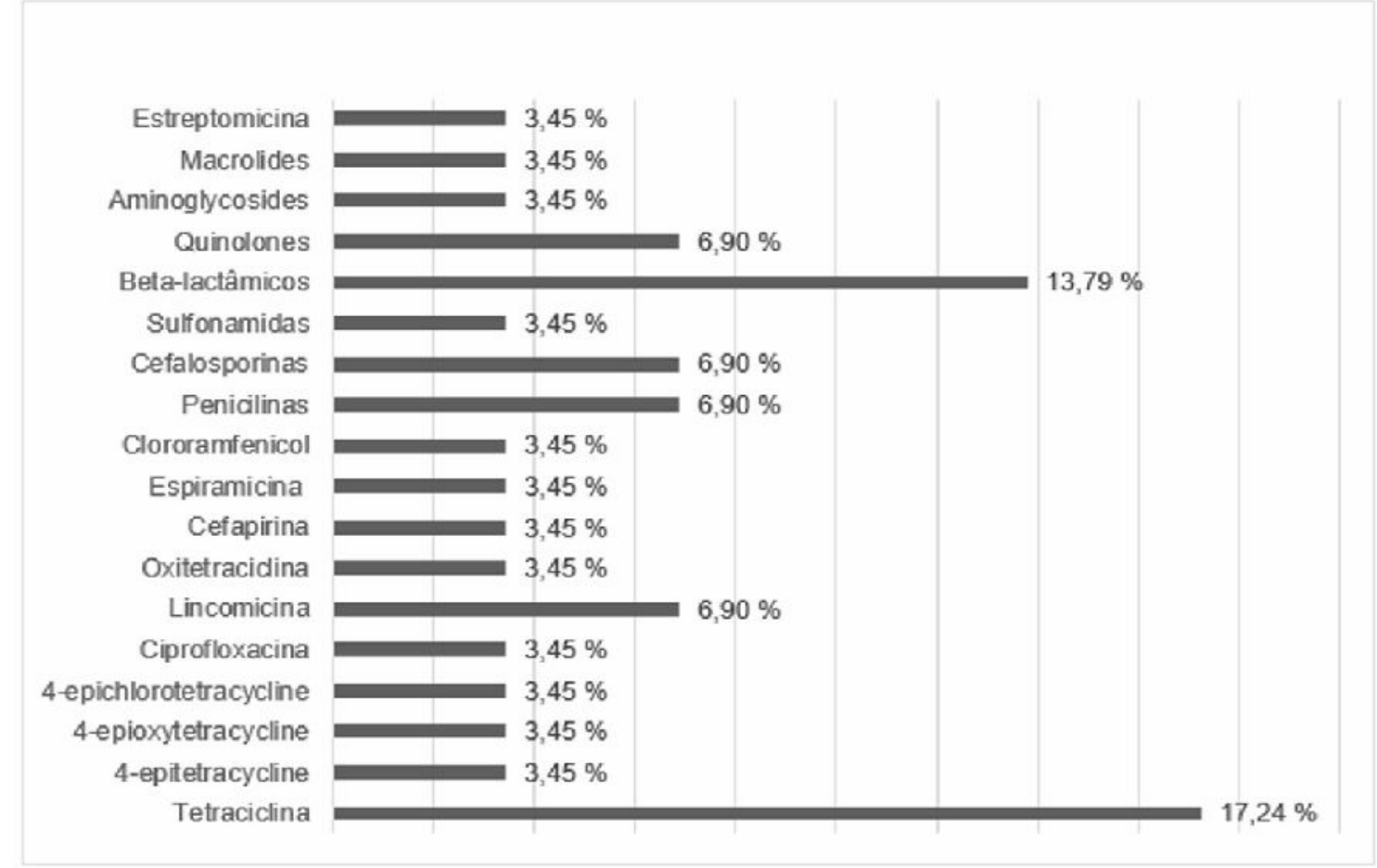

Fonte: Autores (2021). 


\section{Características das Publicações}

$\mathrm{Na}$ Tabela 3 tem-se os respectivos periódicos onde os artigos estudados foram publicados, bem como o número de artigos encontrados, o fator de impacto de cada meio de publicação e sua qualificação Qualis/Capes. Os artigos foram publicados em revistas com fator de impacto alto variando entre 2.534 e 5.548. Estes dados evidenciam a importância dessa problemática para os periódicos indicando estar o foco e escopo dos periódicos de impacto.

O periódico que mais publicou dentro desta temática foi o Food Control que é o jornal científico oficial da Federação Europeia de Ciência e Tecnologia de Alimentos (EFFoST) e da União Internacional de Ciência e Tecnologia de Alimentos (IUFoST) e possui o maior fator de impacto com 5.548, dentre os periódicos da presente revisão sistemática. A Food Control é uma revista internacional que fornece informações essenciais relacionadas ao controle do processo alimentar ou à segurança alimentar de alimentos humanos, dentre às temáticas do periódico aparece claramente a questão foco desta revisão: Segurança alimentar microbiana e sistemas antimicrobianos.

Em relação a qualificação Qualis/Capes, os artigos classificados obtiveram os indicadores mais elevados (A1 e A2), que contemplam periódicos de excelência internacional. Alguns periódicos ainda não foram classificados pelo qualis, por não terem publicações cadastradas na CAPES por pesquisadores brasileiros.

QUADRO 3. Publicações, fator de impacto e Qualis/Capes por periódicos de artigos estudados com o tema resíduos de antimicrobianos em leite animal e derivados.

\begin{tabular}{|c|c|c|c|c|}
\hline \multirow[b]{2}{*}{ Locais publicados } & \multirow{2}{*}{$\begin{array}{c}\text { Números } \\
\text { de } \\
\text { artigos }\end{array}$} & \multirow{2}{*}{$\begin{array}{l}\text { Fator de } \\
\text { impacto }\end{array}$} & \multicolumn{2}{|c|}{ Qualis/Capes } \\
\hline & & & $\begin{array}{l}\text { Ciências de } \\
\text { alimentos }\end{array}$ & $\begin{array}{l}\text { Ciências } \\
\text { Ambientais }\end{array}$ \\
\hline Food Control & 3 & 5.548 & $\mathrm{~A} 1$ & $\mathrm{~A} 1$ \\
\hline $\begin{array}{c}\text { LWT - Food Science and } \\
\text { Technology }\end{array}$ & 1 & 4.952 & NC & NC \\
\hline Journal of Dairy Science & 1 & 3.333 & $\mathrm{~A} 2$ & $\mathrm{~A} 1$ \\
\hline Research in Veterinary & 1 & 2.534 & NC & A2 \\
\hline American Diary Science & 1 & 4.034 & $\mathrm{NC}$ & NC \\
\hline
\end{tabular}

$\mathrm{NC}=$ periódico ainda não classificado pelo sistema Qualis-CAPES; A1 e A2 = contemplam periódicos de excelência internacional.

\section{Triagem de dados}

O Quadro 4 traz as bases de dados aplicadas no estudo, assim como os termos de busca utilizados em cada uma e respectivamente o número de artigos encontrados. Esta análise indicou haver escassez de estudos sobre triagem de antimicrobianos em leite animal e derivados. Mesmo sendo utilizadas palavras de busca em português na pesquisa da base de dados Scielo, não foram encontrados artigos sobre o tema em questão, mostrando o quão importante seria dar enfoque em estudos sobre triagem antimicrobianos em leite animal e derivados. 
QUADRO 4. Artigos sobre resíduos de antimicrobianos em leite animal e derivados, publicados por base de dados e respectivos termos de busca.

\begin{tabular}{|c|c|c|}
\hline Base de dados & Termos de busca & Número de artigos \\
\hline Science Direct & $\begin{array}{c}\text { screening methods, milk, } \\
\text { antibiotic, waste, dairy } \\
\text { products }\end{array}$ & 6 \\
\hline PubMed & $\begin{array}{c}\text { screening methods, milk, } \\
\text { antibiotic, Waste, dairy } \\
\text { products. }\end{array}$ & 1 \\
\hline Scielo & $\begin{array}{c}\text { screening methods, milk, } \\
\text { antibiotic, Waste, dairy } \\
\text { products } \\
\text { métodos de triagem, } \\
\text { antibiótico, leite, resíduos, } \\
\text { produtos lácteos }\end{array}$ & - \\
\hline
\end{tabular}

\section{CONCLUSÃO}

Como foi possível observar nos artigos elencados nesta revisão sistemática, a presença de resíduos de antimicrobianos em leite e seus derivados é uma séria ameaça à saúde pública devido a potencial indução de resistência antimicrobiana, no entanto são poucos os estudos de triagem destas moléculas em produtos comercializados. Conforme evidenciado, os estudos analisados indicam que uma parcela considerável dos alimentos testados está contaminada com resíduos de antibióticos. Entre estes, as tetraciclinas e os $\beta$-lactâmicos são os grupos mais presentes.

Visto a escassez de trabalhos, destaca-se ainda a importância de fomentar e incentivar estudos que investiguem estes aspectos para compreender a real dimensão e direcionar políticas públicas e minimizar os impactos negativos a cadeia de abastecimento alimentar, especialmente no momento atual em que se enfrenta a pandemia de COVID-19. Isso pode chamar a atenção das autoridades de saúde pública para propor planos para mitigar ou interromper essa prática. $E$, dessa forma, limitar a presença de resíduos antimicrobianos em produtos alimentícios de origem animal e o desenvolvimento de resistência antimicrobiana, reduzindo o risco para a saúde pública.

\section{AGRADECIMENTOS}

"O presente trabalho foi realizado com o apoio Coordenação de Aperfeiçoamento de Pessoal de Nível Superior - Brasil (CAPES) - Código de Financiamento 001".

\section{REFERÊNCIAS}

BÄR, C.; Protein profile of dairy products: Simultaneous quantification of twenty bovine milk proteins. International Dairy Journal, [s.I.], p.1-10, jan/2019. Elsevier BV. DOI: http://dx.doi.org/10.1016/j.idairyj.2019.01.001.

BILANDŽIĆ, N.; Veterinary drug residues determination in raw milk in Croatia. Food control, v. 22, n. 12, p. 1941-1948, $2011 . \quad$ DOI: https://doi.org/10.1016/j.foodcont.2011.05.007. 
BRITO M.A.V.P., BRITO J.R.F., SILVA M.A.S. \& CARMO R.A.; Concentração mínima inibitória de dez antimicrobianos para amostras de Staphylococcus aureus isoladas de infecção intramamária bovina. Arquivo Brasileiro Medicina Veterinária e Zootecnia, v. 53, n. 5, p. 531-537, 2001. DOI: https://doi.org/10.1590/S0102-09352001000500003.

DU, B.; Presence of tetracyclines, quinolones, lincomycin and streptomycin in milk. Food Control, v. 100, p. 171-175, 2019. DOI: https://doi.org/10.1016/j.foodcont.2019.01.005.

CHIESA, L.M.; Analysis of antibiotic residues in raw bovine milk and their impact toward food safety and on milk starter cultures in cheese-making process. Lwt, Food Science of Technology v. 131, p. 109783, 2020. DOI: https://doi.org/10.1016/j.Iwt.2020.109783.

CHOWDHURY, R. A review on antibiotics in an animal feed. Bangladesh Journal of Animal Science, v. 38, n. 1-2, p. 22-32, 2012. DOI: https://doi.org/10.3329/bjas.v38i12.9909 .

FERDOUS, J.; Antimicrobial residues in chicken and fish, Chittagong, Bangladesh. EcoHealth, v. 16, n. 3, p. 429-440, 2019. Disponível em: https://link.springer.com/article/10.1007/s10393-019-01430-6.

FONSECA, L. F. L.; SANTOS, M. V. Estratégias para controle de mastites e melhoria da qualidade do leite. Barueri: Manole, v. 1, 2007.

FAO - Food and Agricultrual Organization of the United Nations. Maximum residue limits (MRLs) and risk management recommendations (RMRs) for residues of veterinary drugs in foods, 2018.

GARCIA, S.N.; OSBURN, B.I.; CULLOR, J.S.; A one health perspective on dairy production and dairy food safety. One Health, [s.I.], v. 7, p.100086-00095, jun/ 2019. Elsevier BV. DOI: http://dx.doi.org/10.1016/j.onehlt.2019.100086.

KAZANCOGLU, Y.; OZKAN-OZEN, Y. D.; OZBILTEKIN, M.; Minimizing losses in milk supply chain with sustainability: An example from an emerging economy. Resources, Conservation And Recycling, [s.I.], v. 139, p.270-279, dez/2018. Elsevier BV. DOI:http://dx.doi.org/10.1016/j.resconrec.2018.08.020.

LIBERATI, A.; A declaração PRISMA para relatar revisões sistemáticas e meta-análises de estudos que avaliam intervenções de saúde: explicação e elaboração. Jornal de epidemiologia clínica, v. $62, \quad$ n. 10 , p.e1-e34, 2009. DOI: https://doi.org/10.1016/j.jclinepi.2009.06.006

MARSHALL, B. M.; LEVY, S.B.; Food animals and antimicrobials: impacts on human health. Clinical microbiology reviews, v. 24, n. 4, p. 718-733, 2011. DOI: https://doi.org/10.1128/cmr.00002-11. 
MATRASZEK-ZUCHOWSKA, I.; WOZNIAK, B.; POSYNIAK, A.; Determination of Hormones Residues in Milk by Gas Chromatography-Mass Spectrometry. Food Analytical Methods, [s.I.], v. 10, n. 3, p.727-739, ago/2016. Springer Nature. DOI:http://dx.doi.org/10.1007/s12161-016-0620-5.

MEDEIROS, E.S.; Perfil de sensibilidade microbiana in vitro de linhagens de Staphylococcus spp. isoladas de vacas com mastite subclínica. Pesquisa Veterinária Brasileira [online]. v. 29, n. 7 [Acessado 15 Agosto 2021], p. 569-574, 2009. Disponível em: <https://doi.org/10.1590/S0100-736X2009000700012>.

BRISTY, N.I.; NOMAN, Z.A.; FERDOUS, J.; SACHI, S. KABIR, M.H.; Colistin residue in broiler: Detection in different growth stages. Asian-Australasian Journal of Food Safety and Security, v. 3, p. 43-47, 2019. Disponível em: https://www.sciencedirect.com/science/article/pii/S0924224421000832?via\%3Dihub\#bib 15.

NOYA, I. Environmental and water sustainability of milk production in Northeast Spain. Science Of The Total Environment, [s.I.], v. 616-617, p.1317-1329, mar/ 2018. Elsevier BV. DOI:http://dx.doi.org/10.1016/j.scitotenv.2017.10.186.

NOVAES, S. F. Residues of veterinary drugs in milk in Brazil. Ciência Rural, Santa Maria, v. 47, n. 8, p. 1-7, 2017. DOI: https://doi.org/10.1590/0103-8478cr20170215.

PEREIRA, R. V. Multiresidue screening of milk withheld for sale at dairy farms in central New York State. Journal of dairy science, v. 97, n. 3, p. 1513-1519, 2014. DOI: https://doi.org/10.3168/jds.2013-7421.

POUPAUD, M. Compreender a cadeia de suprimento de antibióticos veterinários para abordar a resistência antimicrobiana na RDP do Laos: Funções e interações das partes interessadas envolvidas. Acta Tropica, v. 220, p. 105943, 2021. DOI: 10.1016 / j.actatropica.2021.105943.

QUINTANILLA, P. Enrofloxacin treatment on dairy goats: Presence of antibiotic in milk and impact of residue on technological process and characteristics of mature $\begin{array}{lllll}\text { cheese. Food } \quad \text { Control, } & \text { v. } 123, & \text { p. } & 107762, & \end{array}$ DOI:https://dx.doi.org/10.1016/j.foodcont.2020.107762.

RANDALL, L. Detection of antibiotic residues and association of cefquinome residues with the occurrence of Extended-Spectrum $\beta$-Lactamase (ESBL)-producing bacteria in waste milk samples from dairy farms in England and Wales in 2011. Research in veterinary science, $\quad$ v. $96, \quad$ n. $1, \quad$ p. 15-24, $2014 . \quad$ DOI: https://doi.org/10.1016/j.rvsc.2013.10.009.

SACHI, S. Antibiotic residues in milk: Past, present, and future. Journal of advanced veterinary and animal research, v. 6, n. 3, p. 315, 2019. DOI: 10.5455 / javar.2019.f350. 
SANI, A. M.;NIKPOOYAN, H.; MOSHIRI, R.; Aflatoxin M1 contamination and antibiotic residue in milk in Khorasan province, Iran. Food and Chemical Toxicology, v. 48, n. 89, p. 2130-2132, 2010. DOI: https://doi.org/10.1016/j.fct.2010.05.015.

TASCI, F.; CANBAY, H.S.; DOGANTURK, M..; Determination of antibiotics and their metabolites in milk by liquid chromatography-tandem mass spectrometry method. Food Control, v. 127, p. 108147, 2021. DOI: https://doi.org/10.1016/j.foodcont.2021.108147.

TEMPINI, P. N. Multidrug residues and antimicrobial resistance patterns in waste milk from dairy farms in Central California. Journal of dairy science, v. 101, n. 9, p. 81108122, 2018. DOI: https://doi.org/10.3168/jds.2018-14398. 